PMMA layer is not used; oxygen or moisture at grain boundaries provides quenching sites. The fast decay is related to trap-assisted recombination at grain boundaries, whereas the slow decay is related to radiative recombination inside the grains (fig. S9) (32).

This proposition was supported by analyzing the change in $\tau$ and $f$ of $\mathrm{MAPbBr}_{3}$ films with varying stoichiometric ratio. As MABr: $\mathrm{PbBr}_{2}$ increased from 1:1 to 1.05:1, the average lifetime $\tau_{\text {avg }}$ gradually increased from 12.2 to $51.0 \mathrm{~ns}$ (table S5). The short $\tau_{\text {avg }}(12.2 \mathrm{~ns})$ in the film with MABr:PbBr ${ }_{2}=1: 1$ originated from the substantial reduction in $\tau_{2}$. This implies that uncoordinated metallic $\mathrm{Pb}$ atoms at grain boundaries inhibit radiative recombination and cause strong nonradiative recombination (fig. S9). The $\mathrm{MAPbBr}_{3}$ films fabricated with $\mathrm{PbBr}_{2}$-rich perovskite solution (MABr: $\left.\mathrm{PbBr}_{2}=1: 1.05\right)$ had a longer lifetime than films with $\mathrm{MABr}: \mathrm{PbBr}_{2}=1: 1$, possibly through $\mathrm{PbBr}_{2}$-induced surface passivation (31). We calculated the average $L_{\mathrm{D}}$ using a model similar to that in a previous report (fig. S10) (33). The films (MABr: $\left.\mathrm{PbBr}_{2}=1.05: 1\right)$ underneath a PMMA layer exhibited a much smaller $L_{\mathrm{D}}(67 \mathrm{~nm})$ than those previously reported $(>1 \mu \mathrm{m})(34)$. We attribute this reduction in $L_{\mathrm{D}}$ to the reduced grain sizes in which excitons are under stronger spatial confinement, thereby reducing dissociation and enhancing radiative recombination; this compensates the plausible adverse effect of larger grain boundary area (6).

The PeLED fabricated from the $\mathrm{MAPbBr}_{3}$ solution (MABr: $\mathrm{PbBr}_{2}=1: 1$ ) without using NCP showed poor luminous characteristics (maximum $\mathrm{CE}=2.03 \times$ $10^{-3} \mathrm{~cd} \mathrm{~A}^{-1}$ ), mainly owing to high leakage current (fig. S11). In contrast, maximum CE was substantially increased $\left(0.183 \mathrm{~cd} \mathrm{~A}^{-1}\right)$ when a fullcoverage uniform $\mathrm{MAPbBr}_{3}$ nanograin layer (MABr:PbBr ${ }_{2}=1: 1$ ) with decreased grain size was achieved with S-NCP, without stoichiometric modifications to avoid metallic $\mathrm{Pb}$ atoms (Fig. 4, $\mathrm{A}$ and $\mathrm{B}$, and Table 1). The maximum $\mathrm{CE}$ was boosted to 21.4 $\mathrm{cd} \mathrm{A}^{-1}$ in the PeLEDs fabricated with perovskite solutions with excess MABr (1.07:1, 1.05:1, 1.03:1 and 1.02:1) (Fig. 4A and Table 1). As $\mathrm{MABr}: \mathrm{PbBr}_{2}$ increased from 1:1 to 1.05:1, the maximum $\mathrm{CE}$ varied from 0.183 to $21.4 \mathrm{~cd} \mathrm{~A}^{-1}$.

We further increased the CE of PeLEDs by using A-NCP. The PeLEDs based on A-NCP had a maximum CE of $42.9 \mathrm{~cd} \mathrm{~A}^{-1}$ (Fig. 4, C and D, and Table 1), which represents an EQE of 8.53\% when the angular emission profile is considered (fig. S12). The EL spectra of PeLEDs were very narrow; full width at half maximum was $\sim 20 \mathrm{~nm}$ for all spectra. This high color purity of OIP emitters shows great potential when used in displays (Fig. 4E). A pixel of the PeLED based on $\mathrm{MABr}: \mathrm{PbBr}_{2}=$ 1.05:1 exhibited strong green-light emission (fig. S13A). Furthermore, the proposed processes and materials used therein are compatible with flexible and large-area devices; a high-brightness flexible PeLED (Fig. 4, F and G) and a large-area (2 cm by $2 \mathrm{~cm}$ pixel) PeLED (fig. S13B) were fabricated. Our study reduces the technical gap between PeLEDs and OLEDs or quantum dot LEDs and is a big step toward the development of efficient next-generation emitters with high color purity and low fabrication cost based on perovskites.

\section{REFERENCES AND NOTES}

W. S. Yang et al., Science 348, 1234-1237 (2015).

2. M. Liu, M. B. Johnston, H. J. Snaith, Nature 501, 395-398 (2013)

3. N. J. Jeon et al., Nature 517, 476-480 (2015)

4. J.-H. Im, I.-H. Jang, N. Pellet, M. Grätzel, N.-G. Park, Nat. Nanotechnol. 9, 927-932 (2014).

5. H. Kim, K.-G. Lim, T.-W. Lee, Energy Environ. Sci. 10.1039/ c5ee02194d (2015)

6. Z.-K. Tan et al., Nat. Nanotechnol. 9, 687-692 (2014).

7. Y.-H. Kim et al., Adv. Mater. 27, 1248-1254 (2015).

8. D. B. Mitzi, Chem. Mater. 8, 791-800 (1996).

9. M. R. Filip, G. E. Eperon, H. J. Snaith, F. Giustino, Nat. Commun 5, 5757 (2014)

10. T. M. Koh et al., J. Phys. Chem. C 118, 16458-16462 (2014)

11. G. E. Eperon et al., Energy Environ. Sci. 7, 982-988 (2014).

12. R. L. Z. Hoye et al., Adv. Mater. 27, 1414-1419 (2015)

13. N. K. Kumawat, A. Dey, K. L. Narasimhan, D. Kabra, ACS Photonics 2, 349-354 (2015).

14. G. Li et al., Nano Lett. 15, 2640-2644 (2015).

15. J. Wang et al., Adv. Mater. 27, 2311-2316 (2015).

16. A. Sadhanala et al., Adv. Electron. Mater. 1, 1500008 (2015).

17. J. C. Yu et al., Adv. Mater. 27, 3492-3500 (2015).

18. N. K. Kumawat et al., ACS Appl. Mater. Interfaces 7 . 13119-13124 (2015)

19. J. H. Noh, S. H. Im, J. H. Heo, T. N. Mandal, S. I. Seok, Nano Lett. 13, 1764-1769 (2013).

20. I. B. Koutselas, L. Ducasse, G. C. Papavassiliou, J. Phys. Condens. Matter 8, 1217-1227 (1996).

21. K. Tanaka et al., Solid State Commun. 127, 619-623 (2003). 22. J. H. Heo, D. H. Song, S. H. Im, Adv. Mater. 26, 8179-8183 (2014).
23. Z. Xiao et al., Adv. Mater. 26, 6503-6509 (2014). 24. N. J. Jeon et al., Nat. Mater. 13, 897-903 (2014). 25. R. Lindblad et al., J. Phys. Chem. C 119, 1818-1825 (2015)

26. E. Dulkeith et al., Nano Lett. 5, 585-589 (2005)

27. S. Gonzalez-Carrero, R. E. Galian, J. Pérez-Prieto, J. Mater. Chem. A 3, 9187-9193 (2015)

28. I. A. Shkrob, T. W. Marin, J. Phys. Chem. Lett. 5, 1066-1071 (2014).

29. P. Schulz et al., Energy Environ. Sci. 7, 1377-1381 (2014).

30. J. Kanbe, H. Onuki, R. Onaka, J. Phys. Soc. Jpn. 43, 1280-1285 (1977)

31. Q. Chen et al., Nano Lett. 14, 4158-4163 (2014).

32. D. Shi et al Science 347, 519-522 (2015).

33. S. D. Stranks et al., Science 342, 341-344 (2013).

34. R. Sheng et al., J. Phys. Chem. C 119, 3545-3549 (2015).

\section{ACKNOWLEDGMENTS}

This work was partially supported by Samsung Research

Funding Center of Samsung Electronics under Project Number SRFC-MA-1402-07. A.S. was partially supported by the Engineering and Physical Sciences Research

Council (UK). All data are available in the main

text and the supplementary materials.

\section{SUPPLEMENTARY MATERIALS}

www.sciencemag.org/content/350/6265/1222/suppl/DC1

Materials and Methods

Supplementary Text

Figs. S1 to S13

Tables S1 to S5

References (35-43)

4 August 2015; accepted 22 October 2015

10.1126/science.aad1818

\title{
Ultraviolet surprise: Efficient soft x-ray high-harmonic generation in multiply ionized plasmas
}

Dimitar Popmintchev, ${ }^{1}$ Carlos Hernández-García, ${ }^{1,2}$ Franklin Dollar, ${ }^{1}$

Christopher Mancuso, ${ }^{1}$ Jose A. Pérez-Hernández, ${ }^{3}$ Ming-Chang Chen, ${ }^{4}$ Amelia Hankla, Xiaohui Gao, ${ }^{5}$ Bonggu Shim, ${ }^{5}$ Alexander L. Gaeta, ${ }^{5}$ Maryam Tarazkar, ${ }^{6}$ Dmitri A. Romanov, ${ }^{7}$ Robert J. Levis, ${ }^{6}$ Jim A. Gaffney, ${ }^{8}$ Mark Foord, ${ }^{8}$ Stephen B. Libby, ${ }^{8}$ Agnieszka Jaron-Becker, ${ }^{1}$ Andreas Becker, ${ }^{1}$ Luis Plaja, ${ }^{2}$ Margaret M. Murnane, ${ }^{1}$ Henry C. Kapteyn, ${ }^{1}$ Tenio Popmintchev ${ }^{1 *}$

High-harmonic generation is a universal response of matter to strong femtosecond laser fields, coherently upconverting light to much shorter wavelengths. Optimizing the conversion of laser light into soft x-rays typically demands a trade-off between two competing factors. Because of reduced quantum diffusion of the radiating electron wave function, the emission from each species is highest when a short-wavelength ultraviolet driving laser is used. However, phase matching-the constructive addition of $\mathrm{x}$-ray waves from a large number of atoms-favors longer-wavelength mid-infrared lasers. We identified a regime of high-harmonic generation driven by 40 -cycle ultraviolet lasers in waveguides that can generate bright beams in the soft x-ray region of the spectrum, up to photon energies of 280 electron volts. Surprisingly, the high ultraviolet refractive indices of both neutral atoms and ions enabled effective phase matching, even in a multiply ionized plasma. We observed harmonics with very narrow linewidths, while calculations show that the $\mathrm{x}$-rays emerge as nearly time-bandwidth-limited pulse trains of $\sim 100$ attoseconds.

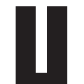
igh-order harmonic generation (HHG) results from the extreme quantum nonlinear response of atoms to intense laser fields: Atoms in the process of being ionized by an intense femtosecond laser pulse coherently emit short-wavelength light that can extend well into the soft $\mathrm{x}$-ray region $(1-6)$. When implemented in a phase-matched geometry to ensure that the laser and HHG fields both propagate at the same speed $\sim c$, HHG from 
many atoms adds constructively to generate bright, coherent beams (7). The high temporal coherence of HHG supports ultrabroad bandwidths $(\Delta \lambda / \lambda \approx 1)$ that simultaneously span many characteristic elemental absorption edges, as well as pulse durations from femtoseconds to attoseconds (8-11), making it possible to capture the fastest electron dynamics in atoms, molecules, nanosystems, and materials (12-23). Moreover, the high spatial coherence of HHG makes it possible to image with spatial resolution near the wavelength limit $(24,25)$. Recently, the use of mid-infrared (IR) femtosecond driving lasers enabled the generation of bright $\mathrm{x}$-ray supercontinua extending to photon energies greater than $1.6 \mathrm{keV}(26)$.

To date, however, generating bright harmonics demanded a trade-off between two competing factors. High harmonics are radiated as a result of a laser-driven quantum-coherent electron recollision process that occurs while an atom is ionizing. This physics dictates that the HHG emission per atom is brightest for short-wavelength ultraviolet (UV) driving lasers, because the short laser cycle minimizes quantum diffusion of the electron wave packet as it propagates away from, and then back to, its parent ion. This maximizes the probability of the recollision HHG event and hence the HHG yield per atom (27). In contrast, generating and phase-matching HHG in the soft $\mathrm{x}$-ray region favors long-wavelength, mid-IR lasers $(>0.8 \mu \mathrm{m})(26,28-30)$. This can be understood from simple arguments. First, the maximum HHG photon energy emitted by a single atom scales quadratically with laser wavelength and is given by $h v_{\mathrm{SA}} \approx I_{\mathrm{p}}+3.17 U_{\mathrm{p}}(4)$, where $U_{\mathrm{p}} \propto I_{\mathrm{L}} \lambda_{\mathrm{L}}{ }^{2}$ is the average quiver energy of the electron in a laser field of intensity $I_{\mathrm{L}}$ and wavelength $\lambda_{\mathrm{L}}$. Second, phase matching is achieved by balancing the positive dispersion of the neutral gas with the negative contribution of the free electrons and is only possible for ionization levels of $<5 \%$ for argon. Fortunately, the lower laser intensity required for long-wavelength driving lasers means that the medium is only weakly ionized, thereby enabling phase matching.

Very weak HHG emission at high photon energies of $\sim 200$ to $500 \mathrm{eV}$ at very low (few torr) gas pressures has been observed using intense UV and near-IR lasers $(1,31,32)$. In those studies, the HHG emission emerged from ionization of a small number of ions, which emit harmonics with comparable efficiency to neutral atoms (33). Moreover, the inability to phase-match the HHG

\footnotetext{
JILA, University of Colorado, Boulder, C0 80309, USA ${ }^{2}$ Grupo de Investigación en Óptica Extrema, Universidad de Salamanca, E-37008 Salamanca, Spain. ${ }^{3}$ Centro de Laseres Pulsados, E-37008 Salamanca, Spain. ${ }^{4}$ Institute of Photonics Technologies, National Tsing Hua University, Hsinchu 30013, Taiwan. ${ }^{5}$ School of Applied and Engineering Physics, Cornell University, Ithaca, NY 14853, USA. ${ }^{6}$ Department of

Chemistry, Temple University, Philadelphia, PA 19122, USA.

${ }^{7}$ Department of Physics, Temple University, Philadelphia, PA 19122, USA. ${ }^{8}$ Physics Division. Physical and Life Sciences, Lawrence Livermore National Laboratory, Livermore, CA 94550, USA.

*Corresponding author. E-mail: tenio.popmintchev@jila.colorado.edu
}

process in plasmas, combined with strong plasmainduced defocusing of the laser, meant that the HHG flux was weaker than theoretically possible in a phase-matched geometry by a factor of $10^{-4}$ to $10^{-11}$.

Here, we demonstrate a regime of bright highharmonic emission in multiply ionized plasmas

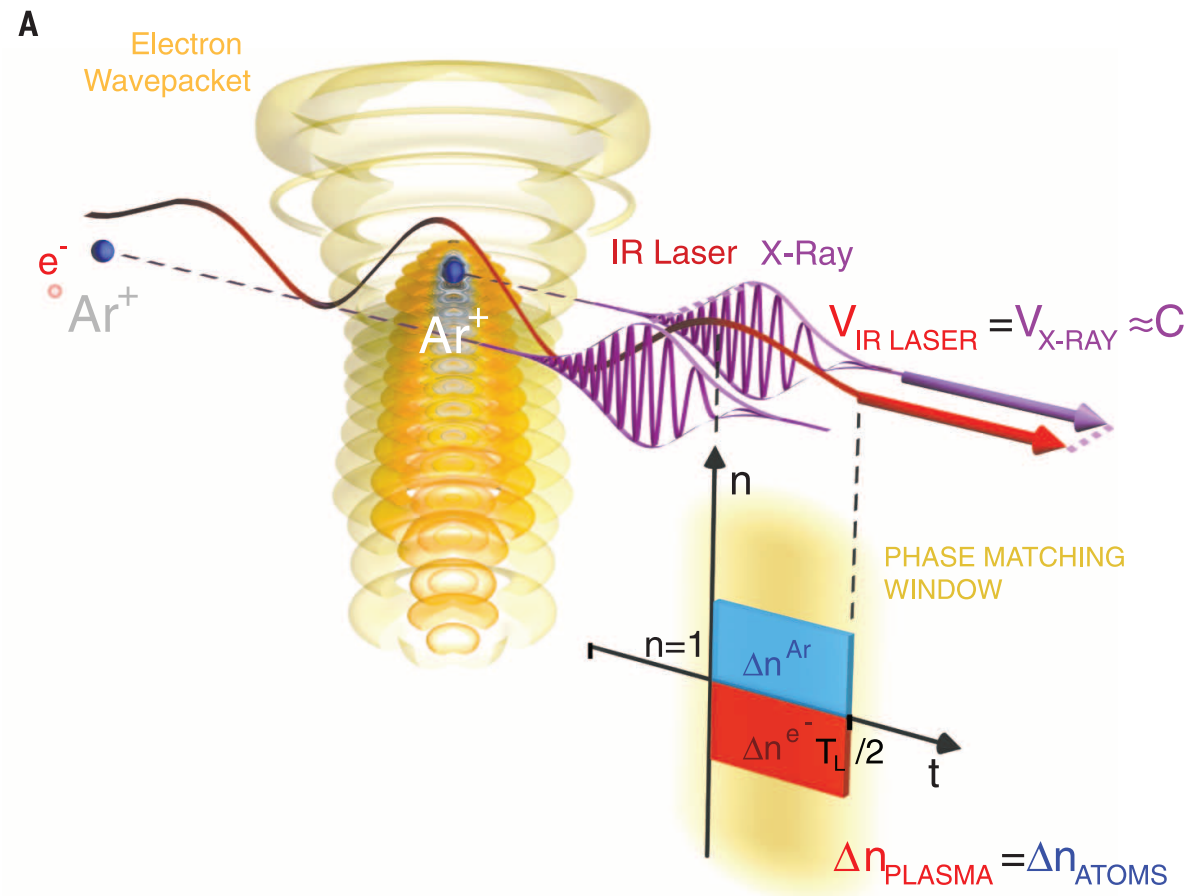

B

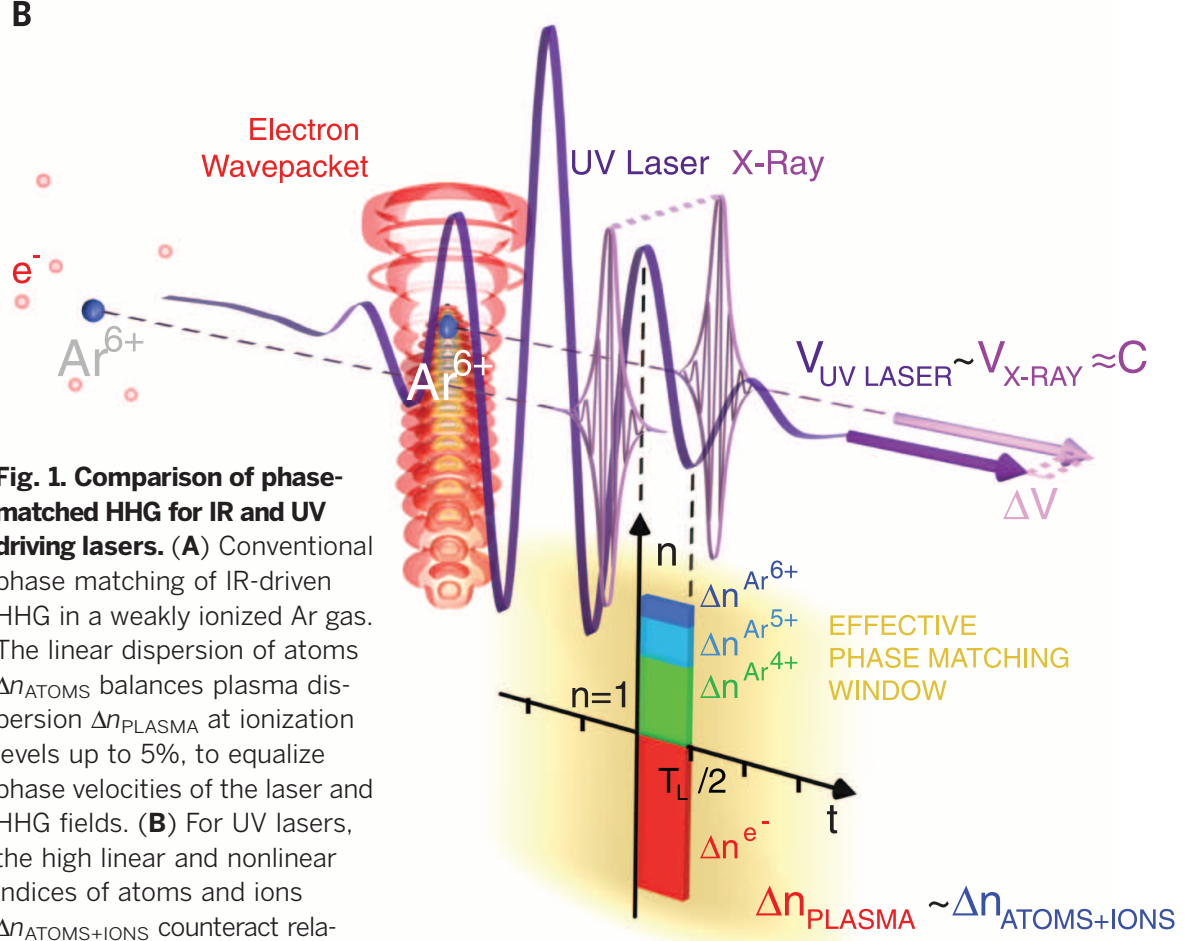

(Figs. 1 and 2). Using intense UV driving lasers at a wavelength of $0.27 \mu \mathrm{m}$, bright discrete $\mathrm{HHG}$ peaks extend well into the soft $x$-ray region of the spectrum. It is well established that low quantum diffusion of the electron wave packet (due to the shorter time the electron spends away from the ion) maximizes the single-atom yield for UV 
driving lasers. However, in this new regime of intense UV-driven HHG, the higher linear and nonlinear indices of atoms and ions contribute substantially to the dispersion experienced by the driving laser and serve to counteract plasma dispersion (Fig. 1B). This enables coherent buildup of the HHG signal in multiply ionized plasmas at very high gas pressures (i.e., effective phase matching) to photon energies greater than $280 \mathrm{eV}$, corresponding to harmonic orders of only $\sim 61$.

We observed high conversion efficiencies of $\sim 10^{-3}$ to $10^{-7}$ throughout the extreme UV (EUV) and soft $\mathrm{x}$-ray regions; these values are orders of magnitude brighter than has been observed to date from ions. The well-separated $(\sim 9 \mathrm{eV})$ narrowband HHG peaks, with linewidths of 70 to $700 \mathrm{meV}(\lambda / \Delta \lambda \approx 350$ to 450$)$ in the vacuum UV (VUV) to soft $x$-ray regions, are ideal for applications in imaging and photoelectron spectroscopies. The high HHG efficiency and narrow linewidths arise from a long phase-matching window as well as low group velocity walk-off, so that group and phase velocity matching are possible over many laser cycles. Moreover, in contrast to the strongly chirped HHG emission driven by near- and mid-IR driving lasers, simulations show that UV-driven harmonics emerge as nearly time-bandwidth-limited series of $\sim 100$-as bursts. Our predictions indicate that bright UVdriven harmonics can extend into the multi-keV region. This leads to the surprising conclusion that the fundamental of Ti:sapphire lasers may be the most limited for generating bright soft $x$-ray harmonics, relative to using longer- or shorter-wavelength driving lasers.

In our experiment, high harmonics were produced by focusing the third harmonic $(0.27 \mu \mathrm{m})$ of a Ti:sapphire laser into an Ar-filled waveguide (diameter 150 to $400 \mu \mathrm{m}$, length 1 to $15 \mathrm{~mm}$ ) at pressures between 1 and 1500 torr (see supplementary materials). The pulse energy and duration were $2.6 \mathrm{~mJ}$ and 35 fs [full width at half maximum (FWHM)], respectively. By focusing to a spot size less than $55 \mu \mathrm{m}$ in diameter, the peak laser intensity was $>6 \times 10^{15} \mathrm{~W} / \mathrm{cm}^{2}$, with a confocal parameter less than $17 \mathrm{~mm}$.

Figure 2A shows that bright HHG emission from $\mathrm{Ar}$ extends far beyond the $\sim 30-\mathrm{eV}$ limit predicted using only the dispersion of atoms, to photon energies of $>280 \mathrm{eV}$ (see fig. S1 for HHG plotted on a linear scale). The high laser intensity required for UV-driven soft x-ray HHG (at $>6 \times 10^{15} \mathrm{~W} / \mathrm{cm}^{2}$ ) is well above the barrier suppression ionization for atoms; thus, neutral atoms will be fully ionized well before the peak intensity of the pulse. However, this intensity is in the tunneling regime for ions. Moreover, time-dependent Schrödinger equation (TDSE) calculations (Fig. 2B and fig. S3) show that HHG from Ar in this energy range must emerge from ions $\left(\mathrm{Ar}^{+}\right.$to $\left.\mathrm{Ar}^{5+}\right)$ with relatively high ionization potentials $\left(I_{\mathrm{p}}^{\mathrm{Ar}^{+}}=27.6 \mathrm{eV}\right.$ to $I_{\mathrm{p}}^{\mathrm{A}^{5+}}=$ $90.1 \mathrm{eV}$ ).

Phase matching in UV-driven VUV and soft x-ray HHG optimizes at higher gas pressures of $\sim 100$ to 400 torr, as shown in Fig. 2A, fig. S1,
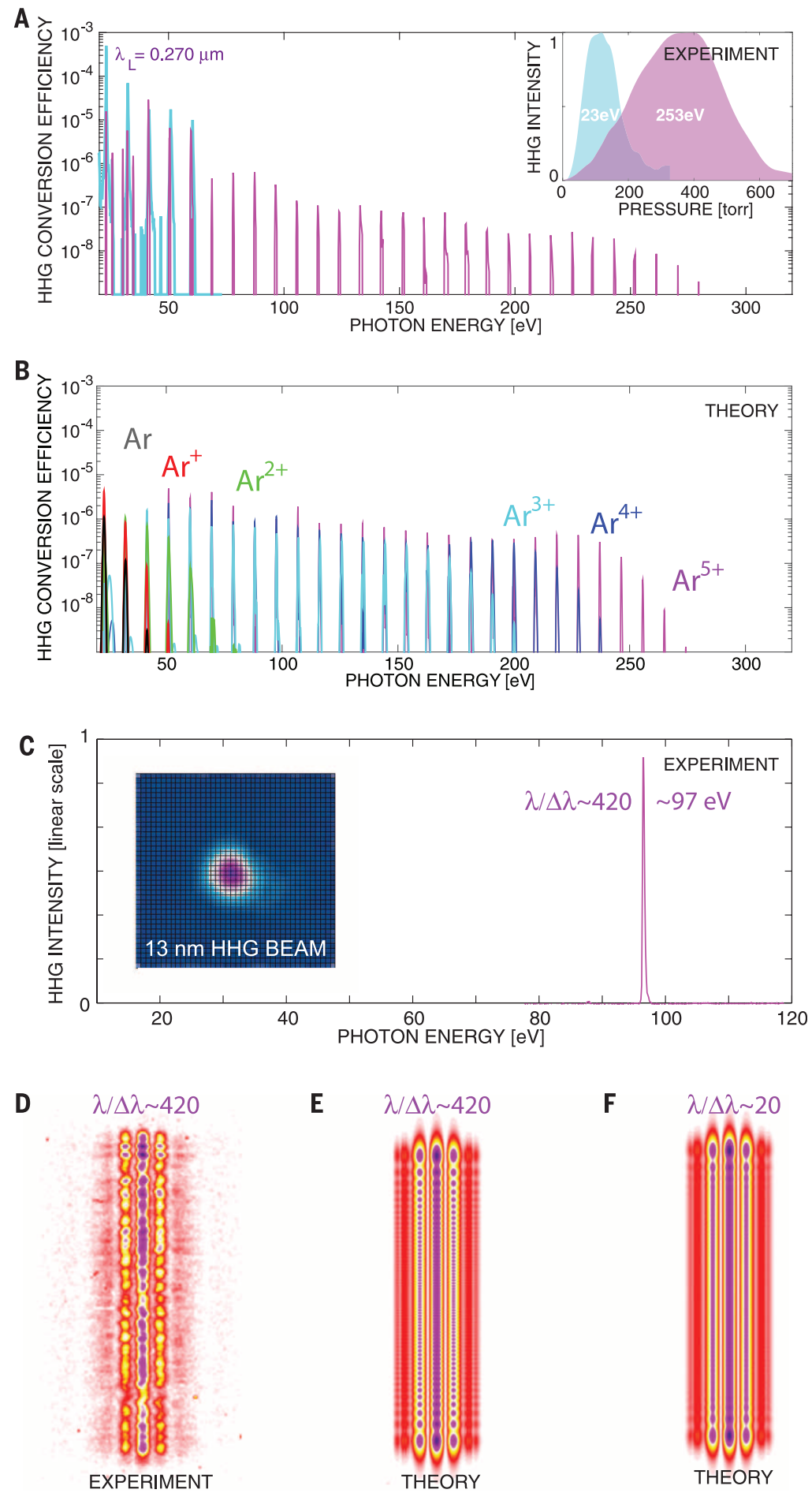

Fig. 2. UV-driven HHG gives bright discrete peaks and isolated harmonics into the soft $\mathrm{x}$-ray region. (A) Experimentally observed UV-driven HHG from multiply ionized Ar, optimized in the EUV (blue spectrum) and soft $x$-ray (purple spectrum) regions. Inset: VUV HHG optimizes at $<100$ torr and low laser intensities $\left(>10^{15} \mathrm{~W} / \mathrm{cm}^{2}\right)$, while soft $x$-ray HHG optimizes at 400 torr and high intensities $\left(>6 \times 10^{15} \mathrm{~W} / \mathrm{cm}^{2}\right)$. (B) Predicted $\mathrm{HHG}$ yield from single Ar atoms and ions using TDSE calculations. Above $30 \mathrm{eV}$, the extended UV-driven HHG must originate from highly charged ions. The emission from different ionic species is plotted separately (not integrated). (C) Experimentally isolated 13-nm harmonic with ultranarrow linewidth of $\lambda / \Delta \lambda \approx 420$ or $230 \mathrm{meV}$. Adjacent harmonic orders were eliminated using Rh $+\mathrm{Si}+$ Be filters. The inset shows the 13-nm HHG beam. (D to F) Experimental and theoretical Young's double-slit diffraction patterns showing full spatial coherence, from a slit of width of $20 \mu \mathrm{m}$ and height $1.5 \mathrm{~mm}$. The spatial modulations perpendicular to the slit in (D) and (E) are due to the high spectral purity that generates observable interferences in (D). For broad harmonic linewidths $(F)$, the vertical spatial modulations are not present. 
A
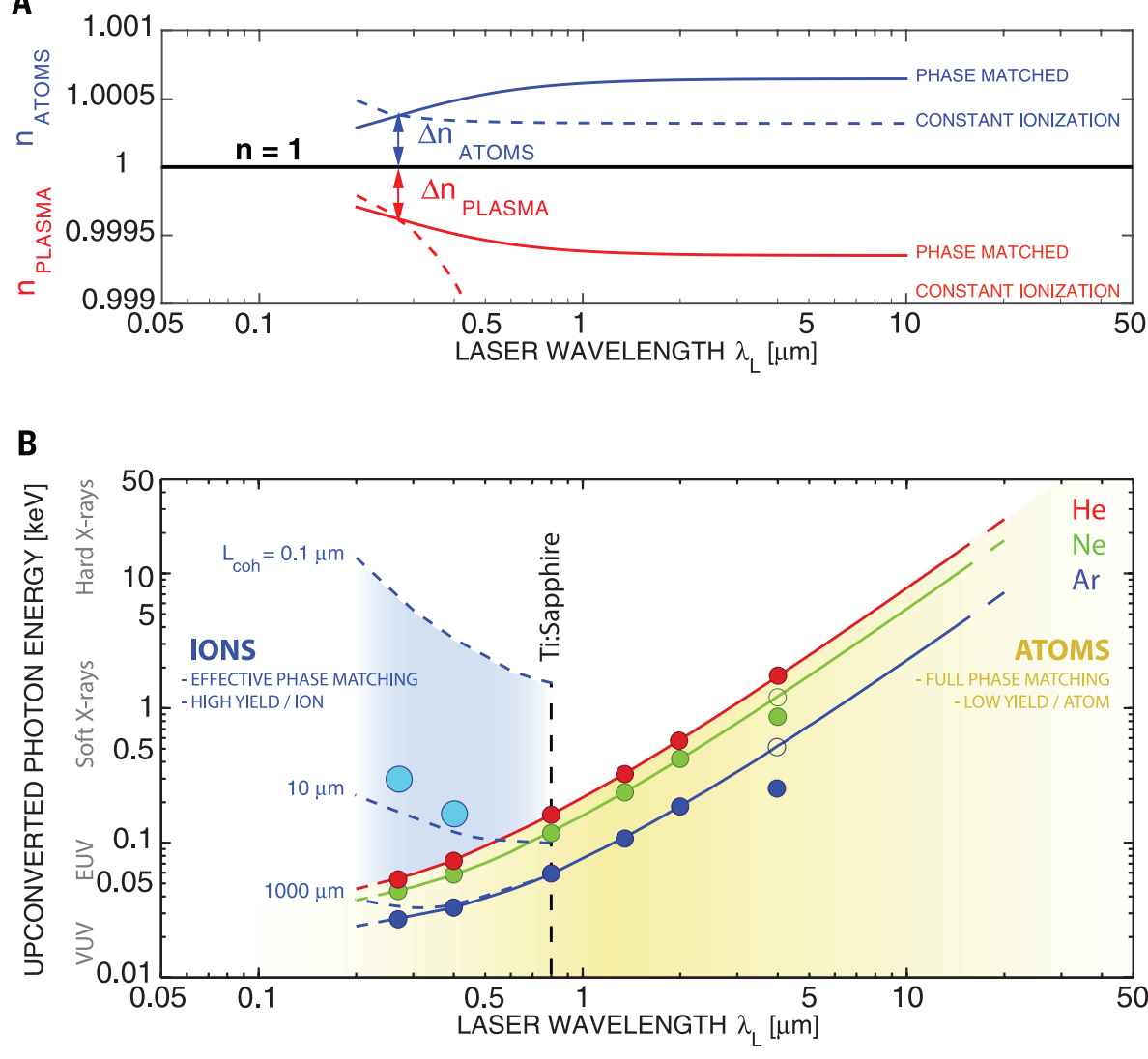

Fig. 3. Phase matching of HHG using UV to mid-IR lasers. (A) Refractive index of Ar atoms (blue dashed) compared with that of free electron plasma (red dashed) as a function of wavelength, for constant ionization conditions of $40 \%$. The solid lines plot the indices under phase-matching conditions near cutoff, where IR-driven HHG needs multi-atmospheres of gently ionized gas, whereas UV-driven HHG occurs in a multiply ionized plasma. (B) Bright HHG from atoms and ions for UV to mid-IR driving lasers. The experimental data are plotted as circles. The solid lines plot the theoretical full phasematching limits, including only the index of neutral atoms. The dashed lines also include the refractive indices of ions, which extend UV-driven HHG effective phase matching into the soft x-ray region.

and table S1. This is in contrast to all previous observations of HHG x-rays from ions in a nonphase-matched regime, where very high harmonic orders can be observed only at pressures that are lower by about two orders of magnitude ( 1 to 3 torr) $(31,33)$. In general, only harmonic orders that are phase-matched will increase in intensity with increasing gas pressure. The optimal pressures increase with harmonic order to compensate for increasing plasma dispersion. Furthermore, the increasing $\mathrm{HHG}$ yield with pressure indicates that ionization-induced defocusing of the laser is not a dominant limitation. Other evidence of good coherent HHG signal buildup over many laser cycles is the narrow spectral width of UVdriven HHG, shown in Figs. 1 and 2 and fig. S1. The linewidths decrease from $>900 \mathrm{meV}$ (at $\sim 50 \mathrm{eV}$ ) for Ti:sapphire lasers, to $\sim 70 \mathrm{meV}$ (at $\sim 30 \mathrm{eV}$ ) and $400 \mathrm{meV}$ (at $\sim 180 \mathrm{eV}$ ) for UV driving lasers. These narrow-linewidth HHG peaks, with spectral purity up to $\lambda / \Delta \lambda>450$, are observed despite strong ionization that must occur on the leading edge of the driving laser pulse and that would normally result in strong spectral broadening and blue shifting of both the driving
At $13 \mathrm{~nm}$, the estimated HHG flux of Fig. 2C corresponds to $>10^{11}$ photons/s (peak brightness $>10^{29}$ photons s${ }^{-1} \mathrm{~mm}^{-2} \mathrm{mrad}^{-2}$ ) at the exit of the waveguide under optimal conditions for generating $280-\mathrm{eV}$ harmonics. Over the whole spectral range, the efficiencies into a single harmonic order are estimated to approach $10^{-3}$ to $10^{-7}$ in the VUV, EUV, and soft x-ray regions. The HHG flux was measured to be consistent using three different methods. First, we compared HHG driven by $0.8-\mu \mathrm{m}$ and $0.27-\mu \mathrm{m}$ lasers in the same spectral region. Second, we estimated the efficiency from known spectrometer, x-ray filter, and charge-coupled device efficiencies. Third, we used a NIST-calibrated vacuum diode to measure the flux in the VUV and EUV regions. We observed good HHG signal-comparable to or greater than phase-matched $\mathrm{HHG}$ at $100 \mathrm{eV}$ driven by $0.8-\mu \mathrm{m}$ lasers-throughout the EUV and soft $\mathrm{x}$-ray regions (Fig. 2 and fig. S1).

High harmonics were first observed in 1987 using a UV driving laser (1). That work used longer pulses (by a factor of $\sim 10$ ) in a tight focus geometry, and HHG up to $85 \mathrm{eV}(14.6 \mathrm{~nm})$ was observed with estimated efficiency of $10^{-11}$. Subsequent work observed HHG from ions (also using long-pulse UV lasers in a tight focus geometry with limited interaction length), with conversion efficiencies around $10^{-10}$ or $10^{-12}$ at photon energies from 100 to $180 \mathrm{eV}$ (32). More recently, broadband HHG from Ar ions up to $500 \mathrm{eV}$ was observed for $0.8-\mu \mathrm{m}$ driving lasers in a few-torr gas-filled hollow waveguide (31). However, this emission was also extremely weak because of the low pressures (few torr) and poor phase-matching conditions (see table S1 for comparison).

To quantitatively explain why bright harmonics from Ar driven by UV lasers can extend into the soft x-ray region, we used simple numerical models, which have been extensively validated for near and mid-IR driving lasers, as well as exact integration of the time-dependent Schrödinger equation $(26,28-30)$. To aid with physical insight, Fig. 3A (dashed lines) plots the calculated index of refraction contribution of an Ar atom compared with that of a free electron. As expected, the refractive indices of neutral atoms are largest in the UV region of the spectrum.

On the other hand, the free electron plasma contribution to the refractive index scales as $n_{\text {plasma }}=\left[\left(1-\omega_{\mathrm{p}}^{2}\right) / \omega_{\text {LASER }}^{2}\right]^{1 / 2}$, where $\omega_{\mathrm{p}}=\left(n_{e} e^{2} /\right.$ $\left.m_{e} \varepsilon_{0}\right)^{1 / 2}$ is the plasma frequency corresponding to an electron density $n_{e}$, and where $e$ and $m_{e}$ are the charge and mass of the electron. Hence, lower frequencies (i.e., longer laser wavelengths) will experience much higher plasma dispersion, and therefore phase matching occurs only at low ionization levels below the critical ionization $(7,36)$ (table S1).

In contrast, for wavelengths in the visible and UV spectral regions, the positive refractive index contribution of a neutral Ar atom exceeds the negative contribution of a free electron. This corresponds to a critical ionization level of $\sim 40 \%$ for UV-driven $\mathrm{HHG}$ at $\sim 40 \mathrm{eV}$. Moreover, the 
A
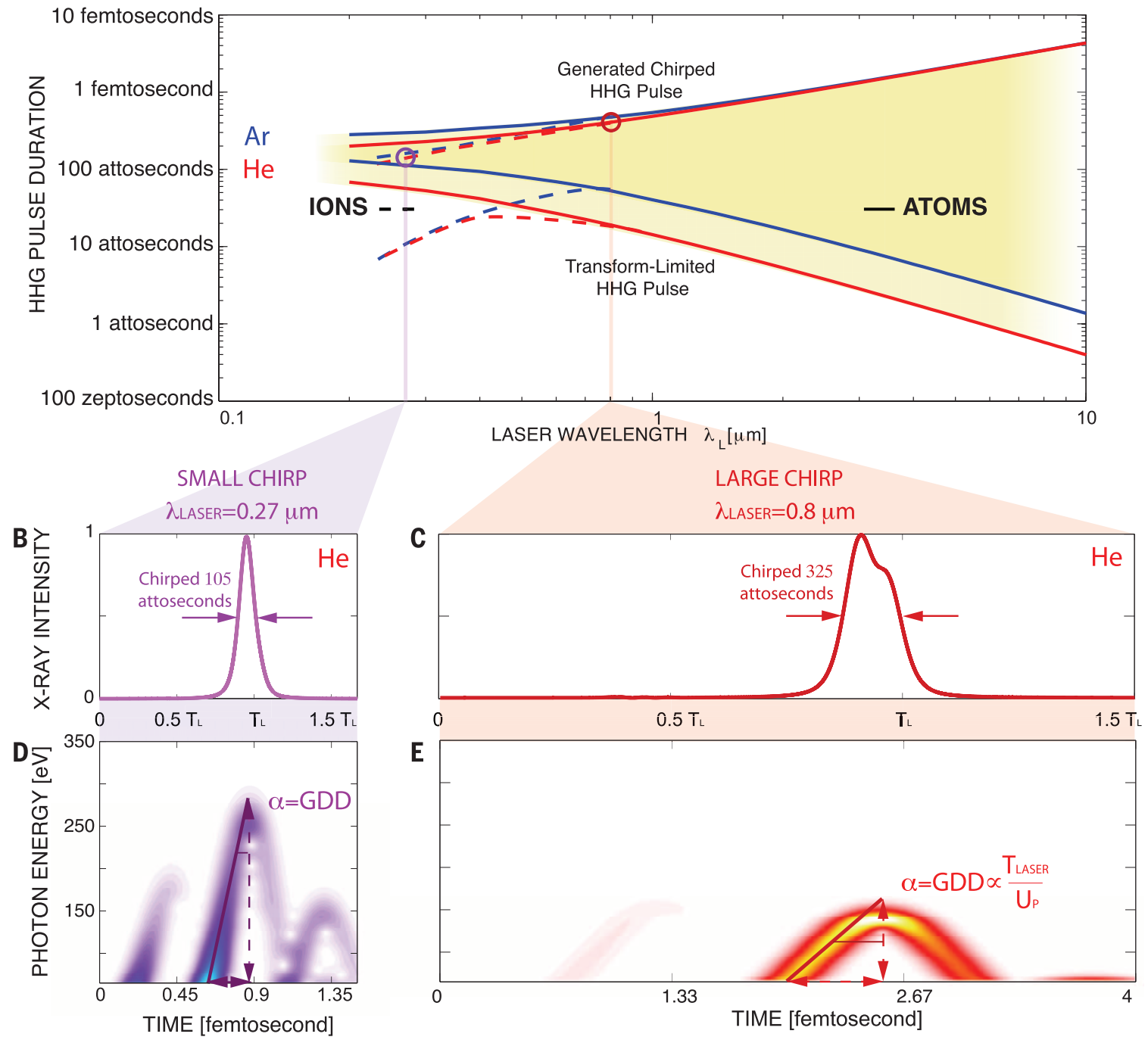

Fig. 4. Theoretical UV-driven HHG gives shorter duration and lower chirp pulses than mid-IR-driven HHG. (A) The pulse duration of HHG soft $x$-ray bursts converges to the transform limit for UV driving lasers. (B to E) Chirp [group delay dispersion (GGD)] of the HHG pulses in He driven by intense three-cycle UV and near-IR lasers, respectively. For UV lasers, the HHG chirp is reduced as a result of the shorter laser cycle duration $T_{\text {LASER }}$ and

the higher ponderomotive potential $U_{P}$ (higher HHG cutoff). The highest harmonics with a $280-\mathrm{eV}$ cutoff (spectrally filtered by a Rh thin film) have a pulse duration of 105 as (even shorter 45- to 75-as pulses could be generated from $\mathrm{He}$ and $\mathrm{He}^{+}$; see supplementary materials). In comparison, for a $0.8-\mu \mathrm{m}$ driver and a phase-matching cutoff of $160 \mathrm{eV}$, the pulse durations are 325 as ( $>3$ times as long).

refractive indices of ions in the UV are comparable to that of neutral $\mathrm{Ar}$ (see supplementary materials), as the ionization potential of each successive ionization stage increases only modestly. Thus, for UV driving lasers, good buildup of the HHG signal is possible even in a multiply ionized plasma. This enables extension of effective phase matching into the soft $x$-ray region and, in theory, even to hard x-rays. Note that for UV-driven HHG, full phase matching is possible in the VUV-EUV, while efficient HHG is possible in soft $\mathrm{x}$-ray region at high pressures, with high photon flux.

In our simple phase-matching model, we consider a one-dimensional geometry, which corresponds to the general case of a large waveguide diameter or large focal spot size (near-plane wave propagation). To calculate the phase mis- match to a first approximation, we need to consider a generalized set of multispecies timedependent equations, given by

$$
\begin{aligned}
& \Delta k(t)=q k_{\omega}(t)-k_{q \omega}(t) \\
& \frac{-q P \eta_{0}(t) \frac{2 \pi}{\lambda_{\mathrm{L}}}\left[\delta_{\mathrm{L}}^{0}(t)+\tilde{n}_{2}^{0}(t) I_{\mathrm{L}}(t)\right]}{\text { neutral atoms }} \\
& \frac{-\sum_{i=1,2, \ldots} q P \eta_{i}(t) \frac{2 \pi}{\lambda_{\mathrm{L}}}\left[\delta_{\mathrm{L}}^{i}(t)+\tilde{n}_{2}^{i}(t) I_{\mathrm{L}}(t)\right]}{\text { multiply charged atoms }} \\
& +\frac{q P \eta_{\text {total }}(t) N_{\text {atm }} r_{e} \lambda_{\mathrm{L}}}{\text { free electrons }}+\Delta k_{\text {geometry }}
\end{aligned}
$$

where $\eta_{i}(t)$ is the fractional population of the various gas species present at a given moment $(i=0$ denotes neutral atoms, $i=1,2,3 \ldots$ denote various ion states), $\eta_{\text {total }}(t)$ is the cumulative fractional ionization [ $\eta_{\text {total }}(t)>100 \%$ for multiply charged ions], $r_{\mathrm{e}}$ is the classical electron radius, $N_{\mathrm{atm}}$ is the number density of atoms at $1 \mathrm{~atm}, P$ is the pressure, and $\delta_{\mathrm{L}}^{i}(t)$ is the difference between the indices of refraction at the fundamental and harmonic wavelengths (which is very large in the UV). Although in general the nonlinear index contributions are estimated to play a rather small role, for high UV laser intensities $>6 \times 10^{15} \mathrm{~W} / \mathrm{cm}^{2}$, an additional term due to the nonlinear index of refraction $(t)$ must also be included, estimated by multiconfigurational self-consistent field calculations (table S2). For $\eta_{\text {total }}(t)>10 \%$, one must include the dispersion of ions as well as a cumulative total ionization level $\eta_{\text {total }}$ resulting from all ions of higher charge. The phase-matching photon energy limits 
are determined by the ionization level at which a dispersion balance is achieved and are independent of HHG geometry (the geometrical dispersion sets only an optimal pressure for a wave-guiding geometry) $(7,29)$. For our experimental conditions, at any time during the HHG emission, two to three species contribute strongly to the HHG signal (Fig. 2B and fig. S2). Fortunately, for a given HHG photon energy, the harmonic order $q$ is lower for UV-driven versus mid-IR-driven HHG, naturally leading to longer coherence lengths even at very high ionization levels and pressures as $\Delta k \propto q$.

Figure S6 plots the estimated dynamic phase mismatch ratio due to plasma and to atoms and ions for Ar driven by 10-cycle 0.27 - and $0.8-\mu \mathrm{m}$ lasers. The dashed black horizontal lines show the high-flux HHG region with coherence lengths $L_{\mathrm{coh}}=\pi / \Delta k$ from infinity to $10 \mu \mathrm{m}$. This time window is substantially longer for UV driving lasers than for near-IR driving lasers, extending effective phase matching over $\sim 5$ to 40 halfcycles or a time interval of $\sim 2$ to 18 fs. This picture is also consistent with the observed narrow spectral linewidths in the VUV to x-ray regime at high phase-matching pressures (Fig. 2 and fig. S1). Under our conditions, at a photon energy of $250 \mathrm{eV}, L_{\mathrm{coh}}>3 \mu \mathrm{m}$, the absorption length $L_{\text {abs }} \approx 1 \mathrm{~mm}$ at 400 torr (for ions see fig. S7), whereas the medium length $L_{\text {med }} \approx 1$ to $15 \mathrm{~mm}$. At $40 \mathrm{eV}, L_{\mathrm{coh}} \approx \infty$.

Additional nonlinear effects-for example, due to laser pulse self-guiding or filamentation, which are likely present-would further enhance effective HHG phase matching in plasmas, because the nonlinear index induced by the laser will increase further to compensate for plasma-induced dispersion. Figure S10 shows blue-shifting and broadening of the fundamental spectrum that is characteristic also for self-effects in efficient HHG at other laser wavelengths, at $0.8 \mu \mathrm{m}$ and in the mid-IR phase-matching regimes $(26,31,37)$. This is also supported by our observation that in a short gas cell geometry the HHG spectrum extends to only $\sim 180 \mathrm{eV}$ in $\mathrm{Ar}$, Ne, or $\mathrm{He}$.

Decreased medium opacity due to removal of several valence electrons can also increase the yield, particularly for intense UV-driven HHG, where the single-atom yield is already very high. However, we calculate that increasing transparency of the medium is not sufficient to explain the observed increase in HHG yield, especially when the coherence length is smaller than the absorption length. As shown in fig. S6, while the transparency increases as expected at photon energies around $100 \mathrm{eV}$, in the soft $\mathrm{x}$-ray region the transparency does not change substantially. Thus, in the future, all-optical quasi-phasematching HHG techniques could be applied to take advantage of the full x-ray absorption length to enhance the yield further (38-41).

We also modeled UV-driven HHG using the TDSE. Figure 2B plots the TDSE predictions for strong field ionization in Ar for a peak laser intensity similar to the experiment, which shows that HHG in the 30- to $280-\mathrm{eV}$ photon energy range must be generated from multiply ionized species, up to $\mathrm{Ar}^{5+}$, at ionization levels above $500 \%$ (on axis of the waveguide). This far exceeds the $5 \%$ critical ionization limit for fully phasematched HHG in Ar using 0.8- $\mu \mathrm{m}$ lasers. Our TDSE calculations show that the single-atom yield scales more strongly than $\sim \lambda_{\mathrm{L}}^{-7.5}$ (42) for a single harmonic of $\mathrm{He}$, corresponding to a factor of $\sim 4000$ greater yield for $0.27-\mu \mathrm{m}$ relative to $0.8-\mu \mathrm{m}$ driving lasers. A similar scaling is expected for Ar species, although with a slightly modified exponent.

These TDSE calculations also show that HHG driven by UV is particularly suitable for generating nearly transform-limited isolated attosecond bursts (or pulse trains), with very low temporal chirp that should be straightforward to compensate. It is already known that the atto-chirp of each individual attosecond burst will be reduced as the driving laser wavelength is reduced in the UV, because of the shorter time the electron spends in the continuum ( 300 as). However, for higher photon energies using more intense UV driving lasers, the pulse duration and atto-chirp will be reduced even further as a result of the larger phase-matched bandwidth (i.e., large $U_{\mathrm{p}}$ ). Figure 4 plots a time-frequency analysis for $\mathrm{HHG}$ in He driven by intense three-cycle UV and $0.8-\mu \mathrm{m}$ lasers, where the reduction in the temporal chirp is very apparent. This effect is dictated by the duration of an individual laser cycle, independent of the number of cycles in the laser pulse. In the case of UV driving lasers, the FWHM duration of the highest harmonics naturally emerges as slightly chirped $~ 105$-as bursts, compared with strongly chirped 325 -as pulses for an $0.8-\mu \mathrm{m}$ driving laser in the same photon energy range. For comparison, for 2- to 4- $\mu \mathrm{m}$ mid-IRdriven HHG, the emission is brightest when the HHG pulse emerges as an isolated, very strongly chirped, 300- to 1200-as burst (26). The experimental $\mathrm{HHG}$ emission from $\mathrm{Ar}$ is predicted to emerge as a long 10-fs train of nearly transformlimited 100-as pulses.

Another advantage of UV driving lasers is the low group velocity walk-off. This is in contrast to mid-IR ( $>2 \mu \mathrm{m}$ )-driven HHG, where group velocity walk-off limits the coherent buildup length and conversion efficiency $(29,43)$. Using UV lasers, the temporal window for phase matching increases while the walk-off decreases (see supplementary materials). Both the group and phase velocities of the driving laser and HHG light can be nearly matched, so that the electron wave packets of different atoms in the medium rescatter in a coherent way (i.e., electron wave packets have the same quantum paths and same quantum phases, resulting in highly temporally coherent HHG). Furthermore, because the coherence length for effective phase matching in the soft $x$-ray region is smaller than the characteristic group velocity walk-off length (see supplementary materials and table $\mathrm{S} 1$ ), intense UV-driven HHG is also effectively group velocitymatched

As shown in Fig. 3B, our best current experimental and theoretical understanding indicates that shorter UV laser wavelengths and intensities $>10^{16} \mathrm{~W} / \mathrm{cm}^{2}$ will produce bright HHG emission at even higher soft x-ray photon energies $>>280 \mathrm{eV}$, which would be ideal for coherent imaging. The phase mismatch decreases more slowly using UV lasers compared with nearIR or mid-IR lasers; this is due to the lower harmonic orders and pressures required (i.e., lower $q$ and $P$ in Eq. 1). To a first approximation under ideal phase-matching conditions, every term in Eq. 1 scales linearly with the laser wavelength. Because lower harmonic orders correspond to much higher photon energies in the case of UV driving lasers, the coherence length is relatively long compared to the coherence length at the same photon energy generated with a longer-wavelength UV-visible laser (Fig. 3B). This ensures strong constructive buildup over the interaction region in an effectively phasematched geometry even in the presence of a finite phase mismatch-and also ensures HHG emission that is as bright as fully phase-matched HHG at $13 \mathrm{~nm}$ in He driven by $0.8-\mu \mathrm{m}$ lasers. This strongly contrasts with longer-wavelength mid-IR lasers, where the order of the upconversion process is higher, with $q>5000$ for a 4- $\mu \mathrm{m}$ driver.

Using intense UV driving lasers, effective phase matching of the HHG process extends the bright emission in the VUV, EUV, and soft x-ray regions. This regime provides coherent light with contrasting and complimentary spectral and temporal properties, compared to the mid-IR-driven HHG x-rays: These exhibit ultrabroad supercontinuum spectra that can be used to probe multiple elements over a spectral range of $>1 \mathrm{keV}$. Remarkably, the UV-driven HHG linewidths decrease to fractional bandwidths of $\lambda / \Delta \lambda \approx 450$ that are ideal for a complementary set of scientific and technological applications, such as in photoemission spectroscopy with very high energy resolution and coherent diffraction imaging with very high spatial resolution $(44,45)$. In terms of laser technology, the availability of intense femtosecond UV lasers (e.g., harmonics of $\mathrm{Yb}$ : YAG, Ti:sapphire, or excimer lasers) makes this approach ideal for many applications. Finally, there is a strong potential to produce much brighter HHG beams at even higher multi-keV xray photon energies than would be possible with other approaches.

\section{REFERENCES AND NOTES}

1. A. McPherson et al., J. Opt. Soc. Am. B 4, 595-601 (1987).

2. M. Y. Kuchiev, JETP Lett. 45, 404-406 (1987).

3. J. L. Krause, K. J. Schafer, K. C. Kulander, Phys. Rev. Lett. 68 3535-3538 (1992)

4. K. C. Kulander, K. J. Schafer, J. L. Krause, in Super-Intense Laser-Atom Physics, B. Piraux, A. L'Huillier, K. Rzazewski, Eds. (Plenum, New York, 1993), pp. 95-110.

5. M. Lewenstein, P. Balcou, M. Y. Ivanov, A. L'Huillier, P. B. Corkum, Phys. Rev. A 49, 2117-2132 (1994).

6. M. Ferray et al., J. Phys. At. Mol. Opt. Phys. 21, L31-L35 (1988)

7. A. Rundquist et al., Science 280, 1412-1415 (1998).

8. I. P. Christov, M. M. Murnane, H. C. Kapteyn, Phys. Rev. Lett. 78, 1251-1254 (1997).

9. P. Antoine et al., Phys. Rev. A 56, 4960-4969 (1997).

10. P. B. Corkum, N. H. Burnett, M. Y. Ivanov, Opt. Lett. 19, 1870 (1994).

11. G. Sansone et al., Science 314, 443-446 (2006). 
12. M. E. Siemens et al., Nat. Mater. 9, 26-30 (2010),

13. S. Mathias et al., Proc. Natl. Acad. Sci. U.S.A. 109, 4792-4797 (2012).

14. E. Turgut et al., Phys. Rev. Lett. 110, 197201 (2013).

15. M. Bauer et al., Phys. Rev. Lett. 87, 025501 (2001).

16. T. Rohwer et al., Nature 471, 490-493 (2011).

17. P. Salieres, A. Maquet, S. Haessler, J. Caillat, R. Taieb, Rep. Prog. Phys. 75, 062401 (2012).

18. R. Haight, Surf. Sci. Rep. 21, 275-325 (1995).

19. M. Probst, R. Haight, Appl. Phys. Lett. 71, 202-204 (1997).

20. A. Rettenberger, P. Leiderer, M. Probst, R. Haight, Phys. Rev. B 56, 12092-12095 (1997)

21. A. L. Cavalieri et al., Nature 449, 1029-1032 (2007).

22. G. Sansone et al., Nature 465, 763-766 (2010)

23. M. Drescher et al., Nature 419, 803-807 (2002).

24. M. D. Seaberg et al., Opt. Express 19, 22470-22479 (2011).

25. J. Miao, T. Ishikawa, I. K. Robinson, M. M. Murnane, Science 348, 530-535 (2015)

26. T. Popmintchev et al., Science 336, 1287-1291 (2012).

27. M. V. Frolov, N. L. Manakov, A. F. Starace, Phys. Rev. Lett. 100 , 173001 (2008)

28. T. Popmintchev et al., Opt. Lett. 33, 2128-2130 (2008).

29. T. Popmintchev et al., Proc. Natl. Acad. Sci. U.S.A. 106 10516-10521 (2009)

30. M. C. Chen et al., Phys. Rev. Lett. 105, 173901 (2010).

31. P. Arpin et al., Phys. Rev. Lett. 103, 143901 (2009).

32. S. G. Preston et al., Phys. Rev. A 53, R31-R34 (1996).
33. E. A. Gibson et al., Phys. Rev. Lett. 92, 033001 (2004).

34. S. C. Rae, Opt. Commun. 104, 330-335 (1994).

35. S. C. Rae, K. Burnett, Phys. Rev. A 46, 1084-1090 (1992).

36. C. G. Durfee et al., Phys. Rev. Lett. 83, 2187-2190 (1999).

37. N. L. Wagner et al., Phys. Rev. Lett. 93, 173902 (2004)

38. X. Zhang et al., Nat. Phys. 3, 270-275 (2007)

39. 0. Cohen et al., Phys. Rev. Lett. 99, 053902 (2007).

40. S. L. Voronov et al., Phys. Rev. Lett. 87, 133902 (2001)

41. P. L. Shkolnikov, A. Lago, A. E. Kaplan, Phys. Rev. A 50, R4461-R4464 (1994).

42. D. Popmintchev et al., in Conference on Lasers and Electrooptics (CLEO) (Optical Society of America, San Jose, CA, 2013), p. QW1A.5.

43. M.-C. Chen et al., Proc. NatI. Acad. Sci. U.S.A. 111. E2361-E2367 (2014).

44. J. Miao, T. Ishikawa, I. K. Robinson, M. M. Murnane, Science $348,530-535$ (2015)

45. B. Zhang et al., Ultramicroscopy 158, 98-104 (2015).

\section{ACKNOWLEDGMENTS}

The experimental work was done at JILA, supported by Army Research Office grant WN11NF-13-1-0259, an NSF PFI AIR award, and U.S. Department of Energy (DOE) grant DE-SC0008803 (M.M.M., T.P., and H.C.K.). Theory was supported by a Marie Curie International Outgoing Fellowship within the EU Seventh Framework Programme for Research

and Technological Development (2007-2013) under REA grant agreement 328334 (C.H.-G.); Junta de Castilla y León (SA116U13, UIC016) and MINECO (FIS2013-44174-P) (C.H.-G. and L.P.): NSF grants PHY-1125844 and PHY-1068706 and AFOSR MURI "Mathematical Modeling and Experimental Validation of Ultrafast Light-Matter Coupling associated with Filamentation in Transparent Media" grant FA9550-10-1-0561 (A.J.-B., R.J.L., X.G., A.L.G., M.M.M., and H.C.K.); Ministry of Science and Technology, Taiwan, grant 102-2112-M-007-025-MY3 (M.-C.C.); U.S. Department of Energy, Division of Chemical Sciences, Atomic, Molecular and Optical Sciences Program (A.B.); and DOE Office of Fusion Energy, HED Laboratory Plasmas program, grant AT5015033 (S.B.L., M.F., and J.A.G.). Lawrence Livermore National Laboratory is operated by Lawrence Livermore National Security LLC for DOE, National Nuclear Security Administration, under contract DE-AC52-07NA27344, LLNL-JRNL-676693. T.P., D.P., M.M.M., and H.C.K. have filed a patent on "Generation of VUV, EUV, X-ray Light Using VUV-UV-VIS Lasers," U.S. patent application 61873794 (2013)/US 20150063385 (2015).

\section{SUPPLEMENTARY MATERIALS}

www.sciencemag.org/content/350/6265/1225/suppI/DC1 Supplementary Text

Figs. S1 to S10

Tables S1 to S3

References (46-65)

7 July 2015; accepted 19 October 2015

10.1126/science.aac9755

\title{
Evidence for a fractional fractal quantum Hall effect in graphene superlattices
}

\author{
Lei Wang, ${ }^{1,2}$ Yuanda Gao, ${ }^{1}$ Bo Wen, ${ }^{3}$ Zheng Han, ${ }^{3}$ Takashi Taniguchi, ${ }^{4}$ \\ Kenji Watanabe, ${ }^{4}$ Mikito Koshino, ${ }^{5}$ James Hone, ${ }^{1}$ Cory R. Dean ${ }^{3 *}$
}

\begin{abstract}
The Hofstadter energy spectrum provides a uniquely tunable system to study emergent topological order in the regime of strong interactions. Previous experiments, however, have been limited to low Bloch band fillings where only the Landau level index plays a role. We report measurements of high-mobility graphene superlattices where the complete unit cell of the Hofstadter spectrum is accessible. We observed coexistence of conventional fractional quantum Hall effect (QHE) states together with the integer QHE states associated with the fractal Hofstadter spectrum. At large magnetic field, we observed signatures of another series of states, which appeared at fractional Bloch filling index. These fractional Bloch band QHE states are not anticipated by existing theoretical pictures and point toward a distinct type of many-body state.
\end{abstract}

$\mathrm{n}$ a two-dimensional electron gas (2DEG) subjected to a magnetic field, the Hall conductivity is generically quantized whenever the Fermi energy lies in a gap (1). The integer quantum Hall effect (IQHE) results from the cyclotron gap that separates the Landau energy levels (LLs). The longitudinal resistance drops to zero, and the Hall conductivity develops plateaus

${ }^{1}$ Department of Mechanical Engineering, Columbia University, New York, NY 10027, USA. ${ }^{2}$ Kavli Institute at Cornell for Nanoscale Science, Ithaca, NY 14853, USA. ${ }^{3}$ Department of Physics, Columbia University, New York, NY 10027, USA. ${ }^{4}$ National Institute for Materials Science, 1-1 Namiki, Tsukuba 305-0044, Japan. ${ }^{5}$ Department of Physics, Tohoku

University, Sendai 980-8578, Japan.

${ }^{*}$ Corresponding author. E-mail: cdean@phys.columbia.edu quantized to $\sigma_{X Y}=v e^{2} / h$, where $v$, the Landau level filling fraction, is an integer; $h$ is Planck's constant; and $e$ is the electron charge. When the 2DEG is modified by a spatially periodic potential, the LLs develop additional subbands separated by minigaps, resulting in the fractal energy diagram known as the Hofstadter butterfly (2). When plotted against normalized magnetic flux $\phi / \phi_{0}$ and normalized density $n / n_{0}$ (representing the magnetic flux quanta and electron density per unit cell of the superlattice, respectively), the fractal minigaps follow linear trajectories (3) according to a Diophantine equation, $n / n_{0}=t \phi / \phi_{0}+s$, where $s$ and $t$ are integers; $s$ is the Bloch bandfilling index associated with the superlattice, and $t$ is a similar index related to the gap structure along the field axis (4) (in the absence of a superlattice, $t$ reduces to the LL filling fraction). The fractal minigaps give rise to QHE features at partial Landau level filling, but in this case $t$, rather than the filling fraction, determines the quantization value $(1,5)$ and the Hall plateaus remain integer-valued.

In very-high-mobility $2 \mathrm{DEGs}$, strong Coulomb interactions can give rise to many-body gapped states also appearing at partial Landau fillings (6-8). Again, the Hall conductivity exhibits a plateau, but in this case quantized to fractions of $e^{2} / h$. This effect, termed the fractional quantum Hall effect (FQHE), represents an example of emergent behavior in which electron interactions give rise to collective excitations with properties fundamentally distinct from the fractal IQHE states. A natural theoretical question arises regarding how interactions manifest in a patterned 2DEG (9-12). In particular, because both the FQHE many-body gaps and the single-particle fractal minigaps can appear at the same filling fraction, it remains unclear whether the FQHE is even possible within the fractal Hofstadter spectrum (13-15). Experimental effort to address this question has been limited, owing to the requirement of imposing a well-ordered superlattice potential while preserving a high carrier mobility (16-18).

Here, we report a low-temperature magnetotransport study of fully encapsulated hexagonal boron nitride (h-BN)/graphene/h-BN heterostructures, fabricated by van der Waals assembly with edge contact $(19,20)$. A key requirement to observe the Hofstadter butterfly is the capability to reach the commensurability condition in which the magnetic length $l_{B}=\sqrt{\hbar / e B}$ (where $\hbar$ is Planck's constant divided by $2 \pi, e$ is the electron charge, and $B$ is the magnetic field) is comparable to the wavelength of the spatially periodic potential, $\lambda$. For experimentally accessible magnetic 


\section{Science}

\section{Ultraviolet surprise: Efficient soft x-ray high-harmonic generation in multiply ionized plasmas}

Dimitar Popmintchev, Carlos Hernández-García, Franklin Dollar, Christopher Mancuso, Jose A. Pérez-Hernández, Ming-Chang Chen, Amelia Hankla, Xiaohui Gao, Bonggu Shim, Alexander L. Gaeta, Maryam Tarazkar, Dmitri A. Romanov, Robert J. Levis, Jim A. Gaffney, Mark Foord, Stephen B. Libby, Agnieszka Jaron-Becker, Andreas Becker, Luis Plaja, Margaret M. Murnane, Henry C. Kapteyn and Tenio Popmintchev

Science 350 (6265), 1225-1231.

DOI: $10.1126 /$ science.aac9755

\section{Short wavelengths birth shorter ones}

The shortest laser pulses--with durations measured in attoseconds--arise from a process termed high-harmonic generation (HHG). Essentially, a longer, "driving" pulse draws electrons out of gaseous atoms like a slingshot, and, when they ricochet back, light emerges at shorter wavelengths. Most HHG has been carried out using light near the

visible/infrared boundary for the driving pulse. Popmintchev et al. used an ultraviolet driving pulse instead, which yielded an unexpectedly efficient outcome. These results could presage a more generally efficient means of creating x-ray pulses for fundamental dynamics studies as well as technological applications. Science, this issue p. 1225

ARTICLE TOOLS

SUPPLEMENTARY MATERIALS

REFERENCES

PERMISSIONS http://science.sciencemag.org/content/350/6265/1225

http://science.sciencemag.org/content/suppl/2015/12/02/350.6265.1225.DC1

This article cites 58 articles, 9 of which you can access for free http://science.sciencemag.org/content/350/6265/1225\#BIBL

http://www.sciencemag.org/help/reprints-and-permissions

Use of this article is subject to the Terms of Service

Science (print ISSN 0036-8075; online ISSN 1095-9203) is published by the American Association for the Advancement of Science, 1200 New York Avenue NW, Washington, DC 20005. The title Science is a registered trademark of AAAS.

Copyright @ 2015, American Association for the Advancement of Science 